\title{
MODES OF TETRAD FORMATION IN TETRASOMIC INHERITANCE
}

\author{
J. A. SVED \\ Department of Genetics, University of Adelaide, South Australia
}

\section{INTRODUCTION}

Received 22.iii.63

THE concept of a mode of gamete formation was introduced by Fisher (1947) as a basic element in extending the theory of linkage from disomic to polysomic inheritance. The similar concept of a mode of tetrad formation (Fisher, 1950) has proved very useful in extending genetical theory to include tetrad material with disomic inheritance, and it is essential for an examination of tetrad formation in tetrasomic organisms.

Fisher (1950) published a formula giving the number of modes of formation of ordered tetrads in terms of the number of linked tetrasomic loci involved. This has been verified by James and Papazian (196r) who also gave the corresponding formula for unordered tetrads. In this article we are concerned with a further discussion and extension. of these enumerations and also with the application of these ideas to the analysis of published data on tetrad segregation for a single locus in autotetraploid yeast.

\section{GENOTYPES AND MODES OF FORMATION OF TETRADS}

The notions of tetrad genotype and mode of tetrad formation have been discussed for disomic inheritance by Bennett (1956). These concepts will be exemplified here for the case of a single tetrasomic locus.

The genetical constitution of any tetrad may be represented by a formula, a typical digenic ordered tetrad formula, for instance, being $\mathrm{AA}, \mathrm{Aa} ; \mathrm{Aa}$, aa, where the semi-colon separates the products of first division of meiosis, and a typical tetragenic unordered tetrad formula, $A_{1} A_{1}, A_{2} A_{3}, A_{3} A_{4}, A_{2} A_{4}$. Tetrads of these formulæ may arise when the parental chromosomes are. AAaa and $\mathrm{A}_{1} \mathrm{~A}_{2} \mathrm{~A}_{3} \mathrm{~A}_{4}$ respectively. Certain permutations of gene symbols within a formula are considered to be of no genetic significance, and tetrads whose formulæ can be produced from each other by these permutations are said to have the same tetrad genotype. For ordered tetrads these permutations are interchange of the chromosomes within the four meiotic products, reversal of the products of first division of meiosis, and reversal of the products of second division, comprising altogether a permutation group of order $2^{7}$. For unordered tetrads a group of permutations of order $2^{4} \cdot 4$ ! is admissible; these permutations are interchange of chromosomes 
within the four meiotic products, and any permutation amongst the meiotic products.

Any two tetragenic genotypes derivable from one another by permutation of the parental chromosomes are said to have the same mode of formation. For example, the two ordered tetrad genotypes $A_{1} A_{1}$, $\mathrm{A}_{3} \mathrm{~A}_{4} ; \mathrm{A}_{2} \mathrm{~A}_{2}, \mathrm{~A}_{3} \mathrm{~A}_{4}$ and $\mathrm{A}_{1} \mathrm{~A}_{1}, \mathrm{~A}_{2} \mathrm{~A}_{4} ; \mathrm{A}_{3} \mathrm{~A}_{3}, \mathrm{~A}_{2} \mathrm{~A}_{4}$ may be produced from each other by interchange of the chromosomes containing the alleles $A_{2}$ and $A_{3}$. The genotypes $A_{1} A_{1}, A_{2} A_{3} ; A_{4} A_{4}, A_{2} A_{3}$, $A_{2} A_{2}, A_{1} A_{4} ; A_{3} A_{3}, A_{1} A_{4}, A_{2} A_{2}, A_{1} A_{3} ; A_{4} A_{4}, A_{1} A_{3}$ and $A_{3} A_{3}, A_{1} A_{2} ;$ $\mathrm{A}_{4} \mathrm{~A}_{4}, \mathrm{~A}_{1} \mathrm{~A}_{2}$ similarly may be derived from each other and from the first pair of genotypes. The mode of tetrad formation by which all these genotypes are produced may be represented conveniently by the first of the six formulæ given, or written for simplicity as I I, 34; 22, 34 . It should be noted that the tetrad genotype as defined above is equivalent to the "quad type" as defined by James and Papazian (I96I), while the mode of tetrad formation corresponds to the "tetrad type " of Leupold (1956b).

The tetrad output from any parental genotype can be completely specified in terms of the frequencies of the modes of formation. These frequencies, which are characteristics of the locus or loci concerned cannot be derived from theoretical consideration but must be estimated from tetrad data. Since all information on segregation and linkage is contained in the estimates of these frequencies their estimation is a primary objective in the analysis of tetrad data.

The analysis of tetrad data is more complex with polysomic than with disomic organisms because of the greatly increased numbers of genotypes and modes of tetrad formation, and also because a tetrad genotype can often be produced from given parental chromosomes by several modes of formation. With disomic material of given constitution, each tetrad genotype may be produced by only one mode of formation which may be inferred directly from the tetrad genotype. The estimation of the mode frequencies is then virtually equivalent to, and follows directly from, the estimation of the tetrad genotype frequencies. With tetrasomic inheritance however, it is only when there are four different alleles at each locus that the mode of formation can be inferred directly from the tetrad genotype. Consequently, when there are only two or three different alleles at a locus, the problem arises of determining how many and which mode frequencies, or independent linear combinations of these frequencies, are estimable from tetrad data with given parental genotypes.

\section{ENUMERATION OF MODES OF TETRAD FORMATION AND TETRAD GENOTYPES}

Fisher's (1950) formula for the number of modes of formation or ordered tetrads in a tetrasomic organism with $l$ linked loci is:

$\frac{\mathrm{r}}{2^{7} 4 !}\left(\begin{array}{r}2520^{l}+4 \cdot 36 \mathrm{O}^{l}+5 \mathrm{I} \cdot 96^{l}+10 \cdot 72^{l}+102 \cdot 4^{8 l}+\mathrm{I} 6 \mathrm{I} \cdot 24^{l}+252^{\cdot} \mathrm{I} 6^{l} \\ +24^{\cdot 1} \cdot 2^{l}+84^{\prime} \cdot 8^{l}+312 \cdot 4^{l}\end{array}\right)$. 
For $l=1,2$ and 3 this gives 8,2538 and 5290732 respectively. The formula given by Fisher and verified by James and Papazian (196I), using essentially the same methods, contains a small printing error in the last term which has been corrected here. For unordered tetrads, the corresponding formula given by James and Papazian is:

$$
\frac{1}{2^{4}(4 !)^{2}}\left(\begin{array}{r}
2520^{l}+4 \cdot 360^{l}+75 \cdot 96^{l}+18 \cdot 72^{l}+150 \cdot 4^{8}+329 \cdot 24^{l}+468 \cdot 16^{l} \\
+72 \cdot 12^{l}+512 \cdot 9^{l}+180 \cdot 8 l+648 \cdot 4^{l}+512 \cdot 3^{l}
\end{array}\right)
$$

giving 5,9 Io and 1767 I 88 when $l=1,2$ and 3 .

TABLE I

Numbers of digenic unordered tetrad formula left unaltered by particular classes of permutations

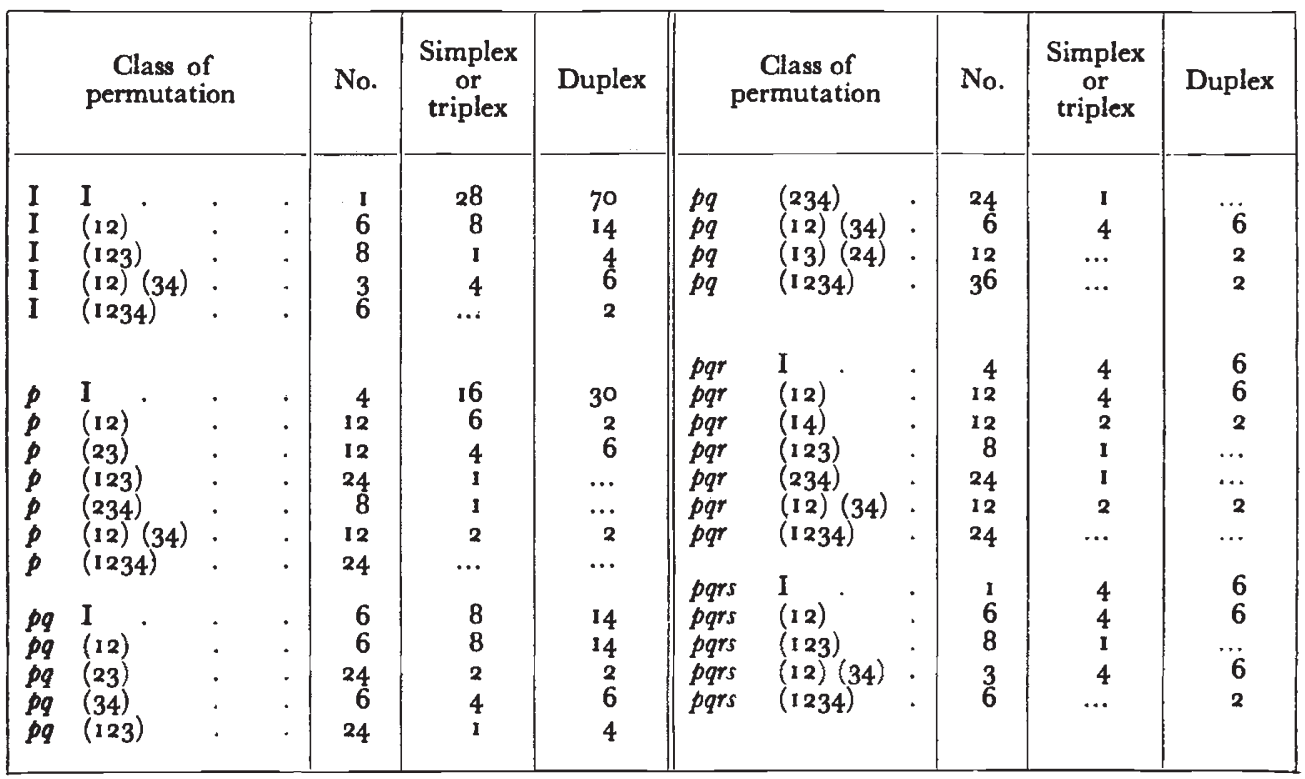

The symbols $p, q, r, s$ represent exchanges of the two chromosomes within each of the four meiotic products. The symbols (12), (123) . . . represent permutations of the products within the unordered tetrad.

Since we would usually be concerned with the analysis of digenic data, the other enumeration of importance is that of the digenic tetrad genotypes. The enumeration of tetrad genotypes, or quad types, from a duplex parental genotype has been given by James and Papazian. Since the simplex and triplex conditions are of the same partitional type (31) we need to consider in addition only one of these since they will give identical enumerations.

Fisher has shown that if at a single locus there are " a " formulæ which are unaltered by a particular permutation, then $a^{l}$ formulæ remain unaltered by this permutation when $l$ linked loci of similar partitional type are considered. The number of genotypes is then $\frac{\mathrm{I}}{\mathrm{N}} \Sigma \mathrm{a}^{l}$ where $\Sigma$ stands for summation over all $\mathrm{N}$ possible permutations. 
For unordered tetrads, the number of formulæ left unaltered by particular permutations are given in table $\mathrm{I}$, considering tetrads arising from digenic parents of all three types at a single locus. For example, considering the permutation $p q$ (123); one simplex formula aa, aa, aa, AA and one triplex formula AA, AA, AA, aa are left unaltered by reversing order in the first two products and permuting the first three products in the manner (123), while four duplex formulæ $\mathrm{Aa}, \mathrm{aA}, \mathrm{Aa}, \mathrm{Aa}, \mathrm{Aa}, \mathrm{aA}, \mathrm{Aa}, \mathrm{aA}, \mathrm{aA}, \mathrm{Aa}, \mathrm{aA}, \mathrm{Aa}$ and $\mathrm{aA}, \mathrm{Aa}, \mathrm{aA}, \mathrm{aA}$ are left unaltered by this procedure. Equivalent permutations from table I have been combined to give table 2 .

TABLE 2

Numbers of formule left unaltered in unordered tetrads

\begin{tabular}{|c|c|c|c|c|c|c|c|c|c|c|c|c|}
\hline \multicolumn{2}{|c|}{ No. of Permutations } & I & 4 & 18 & 12 & 53 & 60 & $3^{2}$ & 96 & 60 & $4^{8}$ & $2^{4} \cdot 4 !$ \\
\hline Simplex parent & . & 28 & 16 & 8 & 6 & 4 & 2 & I & I & $\cdots$ & $\cdots$ & \\
\hline Duplex parent & - & 70 & $3^{\circ}$ & 14 & 2 & 6 & 2 & 4 & $\cdots$ & 2 & $\cdots$ & \\
\hline Triplex parent & . & 28 & 16 & 8 & 6 & 4 & 2 & I & I & $\cdots$ & $\cdots$ & \\
\hline
\end{tabular}

Applying Fisher's theorem, the number of unordered tetrad genotypes from a tetraploid organism duplex at $l$ loci may be derived from table 2 as

$$
\frac{1}{2^{4} 4 !}\left(70^{l}+4 \cdot 30^{l}+18 \cdot 14^{l}+12 \cdot 2^{l}+53 \cdot 6^{l}+60 \cdot 2^{l}+32 \cdot 4^{l}+60 \cdot 2^{l}\right)
$$

which is the formula from table 6 of James and Papazian. Similarly the numbers of tetrad genotypes for an organism simplex at $l$ loci or triplex at $l$ loci is equal to

$$
\frac{\mathrm{I}}{2^{4} 4 !}\left(2^{l}+4 \cdot 16^{l}+18 \cdot 8^{l}+12 \cdot 6^{l}+53 \cdot 4^{l}+60 \cdot 2^{l}+128\right) \text {. }
$$

It follows ( $c f$. Fisher, 1962) by a slight extension of the theorem given by Fisher (1950) that if $a_{1}$ formulæ of one type, $a_{2}$ formulæ of another, and $a_{3}$ of a third are unaltered by a particular permutation and $l_{1}, l_{2}$ and $l_{3}$ linked loci respectively of the three types are considered, then the number of genotypes is given by $\frac{1}{N} \sum a_{1}{ }^{l_{1}} a_{2}{ }^{l_{2}} a_{3}{ }^{l_{3}}$. Thus in the present case, considering a parent simplex at $l_{1}$ loci, duplex at $l_{2}$ and triplex at $l_{3}$, where $l=l_{1}+l_{2}+l_{3}$, the number of unordered tetrad genotypes is given by:

$$
\begin{aligned}
& \frac{\mathrm{I}}{2^{4} 4 !}\left(28^{l_{1}} \cdot 7 \mathrm{0}^{l_{2} \cdot 28^{l_{3}}}+4 \cdot 16^{l_{1}} \cdot 3 \mathrm{O}^{l_{1} \cdot 1} \cdot 6^{l_{3}}+18 \cdot 8^{l_{1}} \cdot 14^{l_{2} \cdot 88^{l_{3}}}\right. \\
& +12 \cdot 6^{l_{1}} \cdot 2^{l_{3}} \cdot 6^{l_{3}}+53 \cdot 4^{l_{1}} \cdot 6^{l_{2}} \cdot 4^{l_{3}}+60 \cdot 2^{l_{1}} \cdot 2^{l_{3}} \cdot 2^{l_{3}}
\end{aligned}
$$

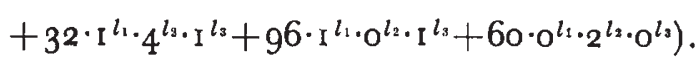


Since there are $\frac{l !}{l_{1} ! l_{2} ! l_{3} !}$ ways in which a parent can be simplex at $l_{1}$ loci, duplex at $l_{2}$ and triplex at $l_{3}$, the total number of unordered tetrad genotypes arising from all possible types of digenic parents is

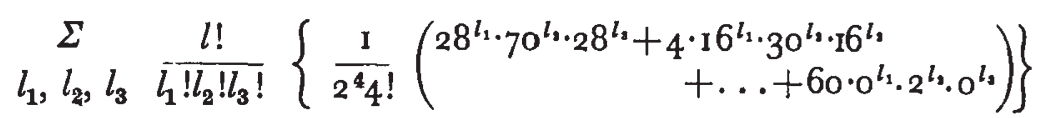

where the summation extends over all possible values of $l_{1}, l_{2}$ and $l_{3}$ conditional on $l_{1}+l_{2}+l_{3}=l$. Simplifying the above expression we get:

$$
\begin{gathered}
\frac{1}{2^{4} 4 !}\left(\sum \frac{l !}{l_{1} ! l_{2} ! l_{3} !} 28^{l_{1} \cdot 20^{l_{1}} \cdot 28^{l_{3}}+4} \Sigma \frac{l !}{l_{1} ! l_{2} ! l_{3} !} \mathrm{I}^{l_{1}} \cdot 30^{l_{1} \cdot 16^{l_{1}}}\right. \\
\left.+\ldots+60 \Sigma \frac{l !}{l_{1} ! l_{2} ! l_{3} !} \mathrm{o}^{l_{1} \cdot 2^{l_{1}} \cdot 0^{l_{3}}}\right) \\
=\frac{1}{2^{4} 4 !}\left((28+70+28)^{l}+4(16+30+16)^{l}+\ldots+60(0+2+0)^{l}\right) \\
=\frac{1}{2^{4} 4 !}\left(126^{l}+4 \cdot 62^{l}+18 \cdot 30^{l}+65 \cdot 14^{l}+92 \cdot 6^{l}+156 \cdot 2^{l}\right) \\
7,167,9477 \ldots
\end{gathered}
$$

\begin{tabular}{|c|c|c|c|c|c|c|c|c|c|c|c|c|}
\hline \multicolumn{4}{|c|}{ No. of permutations } & I & 4 & Io & 4 & 29 & $3^{6}$ & 28 & 16 & $2^{2}$ \\
\hline Simplex or triplex parents & & & & 28 & 16 & 8 & 6 & 4 & 2 & $\cdots$ & $\ldots$ & \\
\hline Duplex parent & - & - & · & 70 & 30 & 14 & 2 & 6 & 2 & 2 & $\cdots$ & \\
\hline Total & . & . & . & 126 & 62 & 30 & 14 & 14 & 6 & 2 & $\cdots$ & \\
\hline
\end{tabular}

Thus the total number of unordered tetrad genotypes which can arise from all possible digenic parents is, as expected, the number

TABLE 3

Numbers of formula left unaltered in ordered tetrads

which would be obtained from table 2 considering invariant simplex, duplex and triplex formulæ. The equivalent calculations for ordered tetrad genotypes are summarised in table 3 , the total number of genotypes being obtained as

$$
\begin{array}{r}
\frac{1}{2^{7}}\left(126^{l}+4 \cdot 62^{l}+10 \cdot 30^{l}+33 \cdot 14^{l}+36 \cdot 6^{l}+28 \cdot 2^{l}\right) \\
\text { II }, 376,25955 \ldots
\end{array}
$$

\section{ANALYSIS FOR A SINGLE LOCUS}

(i) Unordered tetrads

(a) Digenic parental genotype. As previously mentioned, the mode of formation by which a tetrad genotype has arisen cannot be determined 
uniquely in this case. The law of inheritance can be expressed in the form of a tetrad matrix, analogous to the gametic matrix of Fisher (1947).

Tetrad matrices may be constructed for simplex, duplex and triplex parental genotypes, but because of the equivalent partitional form of the simplex and triplex genotypes the matrices for these two genotypes will be identical. It is adequate therefore to treat data from a triplex parent together with data from simplex parents. This may be done by permuting the two allele symbols in the triplex tetrad genotype formulae, generating an equivalent set of formulæ which may be considered as arising from a simplex parental genotype. This argument holds only if there are no viability differences associated with the gene substitution, which of course must be tested each time before the

TABLE 4

Unordered tetrad matrix for digenic parental genotypes

\begin{tabular}{|c|c|c|c|c|c|}
\hline \multirow{2}{*}{$\begin{array}{l}\text { Parental genotypes } \\
\text { Tetrad } \\
\text { tetrad formatypes }\end{array}$} & \multicolumn{2}{|c|}{ Simplex (Aaaa) } & \multicolumn{3}{|c|}{ Duplex (AAaa) } \\
\hline & $\mathrm{AA}, \mathrm{aa}, \mathrm{aa}, \mathrm{aa}$ & $\mathrm{Aa}, \mathrm{Aa}, \mathrm{aa}, \mathrm{aa}$ & $\mathrm{AA}, \mathrm{AA}, \mathrm{aa}, \mathrm{aa}$ & $\mathrm{AA}, \mathrm{Aa}, \mathrm{Aa}, \mathrm{aa}$ & $\mathrm{Aa}, \mathrm{Aa}, \mathrm{Aa}, \mathrm{Aa}$ \\
\hline $\begin{array}{l}\text { I } 11,22,33,44 . \\
\text { II } 11,22,34,34 . \\
\text { III } 12,12,34,34 . \\
\text { IV } 11,23,34,24 \\
\text { V } 13,14,23,24 .\end{array}$ & $\begin{array}{r}12 \\
6 \\
\cdots \\
3 \\
\cdots\end{array}$ & $\begin{array}{r}\dddot{6} \\
12 \\
9 \\
12\end{array}$ & $\begin{array}{r}12 \\
4 \\
4 \\
\cdots \\
\cdots\end{array}$ & $\begin{array}{r}\dddot{8} \\
\ldots \\
12 \\
8\end{array}$ & $\begin{array}{c}\cdots \\
\cdots \\
3 \\
\cdots \\
4\end{array}$ \\
\hline
\end{tabular}

simplex and triplex data can be pooled. The process is illustrated by a numerical example in section 5 .

The tetrad matrix, or in this case the combined matrix for simplex and duplex genotypes is constructed by considering the array of tetrad genotypes produced by each mode of formation from the simplex and duplex parents in turn. For example the mode, I2, I2, 34, 34 produces from the parental chromosomes A, A, a, a one third of the tetrad genotype AA, AA, aa, aa and two thirds of the genotype Aa, Aa, Aa, Aa. This may be deduced 'most expeditiously by writing for the alleles $A_{1}, A_{2}, A_{3}$ and $A_{4}$ in turn $A, A, a, a, A, a, A, a, A, a, a, A, a, A, A, a$, a, $A, a, A$ and $a, a, A, A$, each of the six combinations being equally likely. Combinations I and 6 yield the genotype AA, AA, aa, aa when the appropriate substitutions for $A_{1} A_{2} A_{3}$ and $A_{4}$ in terms of $A$ and $a$ are made in the mode formula, $A_{1} A_{2}, A_{1} A_{2}, A_{3} A_{4}, A_{3} A_{4}$, while combinations 2, 3, 4 and 5 yield the genotype $\mathrm{Aa}, \mathrm{Aa}, \mathrm{Aa}, \mathrm{Aa}$. The completed tetrad matrix is given in table 4 .

If the frequencies of the five modes of tetrad formation are $f_{1} \ldots$ $f_{5}$ so that $f_{1}+\ldots+f_{5}=\mathrm{I}$, and the expected frequencies of the five tetrad genotypes are $m_{1} \ldots m_{5}$, with the restrictions that $m_{1}+m_{2}=\mathrm{I}$ 
and $m_{3}+m_{4}+m_{5}=\mathrm{I}$, the tetrad matrix is equivalent to a set of five equations:

$$
\begin{aligned}
\mathrm{I} 2 f_{1}+6 f_{2}+3 f_{4} & =\mathrm{I} 2 m_{1} \\
6 f_{2}+\mathrm{I} 2 f_{3}+9 f_{4}+\mathrm{I} 2 f_{5} & =\mathrm{I} 2 m_{2} \\
\mathrm{I} 2 f_{1}+4 f_{2}+4 f_{3} & =\mathrm{I} 2 m_{3} \\
8 f_{2} \quad \mathrm{I} 2 f_{4}+8 f_{5} & =\mathrm{I} 2 m_{4} \\
8 f_{3}+4 f_{5} & =\mathrm{I} 2 m_{5}
\end{aligned}
$$

The notation used here corresponds to that of Fisher (1947) and Griffing (1957). In what follows, it can be shown that the restrictions on the genotype and mode frequencies may be ignored without affecting the argument.

What is required from the above set of equations is the solution

\begin{tabular}{|c|c|c|c|c|}
\hline \multicolumn{5}{|c|}{ Mode of tetrad formation } \\
\hline I & II & III & IV & $\mathrm{V}$ \\
\hline 1 & -3 & $\ldots$ & 2 & .. \\
\hline$\ldots$ & 1 & -1 & -2 & 2 \\
\hline
\end{tabular}
for $f_{1} \ldots f_{5}$ in terms of $m_{1} \ldots m_{5}$. Such a solution is only possible in the case where the number of independent equations is equal to or greater

TABLE 5

Deficiency matrix for digenic unordered tetrads

than the number of unknowns. However by subtracting suitable multiples of one row of the matrix from another, and repeating the process till as many rows as possible are made equal to zero, we can show that in this case a maximum of two rows can be made equal to zero. This can be shown as equivalent to the statement that only three of the above equations are independent, the left hand sides of the other two equations being expressible as linear combinations of these three.

Corresponding to each of the zero rows, a linear combination of the mode frequencies is obtained, having the property that any multiple of either of the two combinations may be added to the five mode frequencies without affecting the tetrad genotype output. In the present case, the two linear combinations are $f_{1}-3 f_{2}+2 f_{4}$ and $f_{2}-f_{3}-2 f_{4}+2 f_{5}$, or any two independent linear combinations of these two. Expressing these in the form of a two row "deficiency matrix" gives the result as shown in table 5 .

It can be shown that any estimable linear combination of the mode frequencies is one which when written in column vector form, postmultiplies the deficiency matrix to zero. Three of the simplest estimable combinations adding to unity are in this case $u_{1}=f_{5}+2 f_{3}$, 
$u_{2}=f_{4}-2 f_{1}-2 f_{3}, u_{3}=f_{2}+3 f_{1}+f_{3}$. Since the mode frequencies, $f_{5}, f_{4}$ and $f_{2}$ each appear only once, in the combinations $u_{1}, u_{2}$ and $u_{3}$ respectively, it follows that the matrix connecting the frequencies of the three linear combinations to the tetrad genotype frequencies

TABLE 6

Reduced unordered tetrad matrix for digenic parental genotypes

\begin{tabular}{|c|c|c|c|c|c|}
\hline $\begin{array}{l}\text { Frequencies } \\
\text { of estimable } \\
\text { linear combinations }\end{array}$ & $m_{1}$ & $m_{2}$ & $m_{3}$ & $m_{4}$ & $m_{8}$ \\
\hline$u_{1}=f_{5}+2 f_{3}$ & $\ldots$ & 12 & $\ldots$ & 8 & 4 \\
\hline$u_{3}=f_{4}-2 f_{1}-2 f_{8}$ & 3 & 9 & $\cdots$ & 12 & $\cdots$ \\
\hline$u_{3}=f_{2}+3 f_{1}+f_{3}$ & 6 & 6 & 4 & 8 & $\ldots$ \\
\hline
\end{tabular}

must have as its first, second and third rows the fifth, fourth and second rows respectively of the original matrix. The completed matrix as given in table 6 then represents a set of five equations in three

TABLE 7

Unordered tetrad matrix for trigenic parental genotype $A A^{\prime} a a$

\begin{tabular}{|c|c|c|c|c|c|}
\hline Genotype & $\mathrm{AA}, \mathrm{A}^{\prime} \mathrm{A}^{\prime}, \mathrm{aa}, \mathrm{aa}$ & $\mathrm{AA}^{\prime}, \mathrm{AA}^{\prime}, \mathrm{aa}, \mathrm{aa}$ & $\begin{array}{l}\mathrm{AA}, \mathrm{A}^{\prime} \mathrm{a}, \mathrm{A}^{\prime} \mathrm{a}, \mathrm{aa} \\
\mathbf{a}^{+} \\
\mathrm{A}^{\prime} \mathrm{A}^{\prime}, \mathrm{Aa}, \mathrm{Aa}, \mathrm{aa}\end{array}$ & $\mathrm{AA}^{\prime}, \mathrm{Aa}, \mathrm{A}^{\prime} \mathrm{a}, \mathrm{aa}$ & $\mathrm{Aa}, \mathrm{Aa}, \mathrm{A}^{\prime} \mathrm{a}, \mathrm{A}^{\prime} \mathrm{z}$ \\
\hline $\begin{array}{l}\text { I } \\
\text { II } \\
\text { III } \\
\text { IV } \\
\text { V }\end{array}$ & $\begin{array}{l}6 \\
1 \\
\cdots \\
\cdots \\
\cdots\end{array}$ & $\begin{array}{c}\cdots \\
1 \\
2 \\
\cdots \\
\cdots\end{array}$ & $\begin{array}{r}\ldots \\
4 \\
\ldots \\
3 \\
\ldots\end{array}$ & $\begin{array}{l}\ldots \\
\cdots \\
\cdots \\
3 \\
4\end{array}$ & $\begin{array}{r}\ldots \\
\ldots \\
4 \\
\cdots \\
2\end{array}$ \\
\hline
\end{tabular}

Deficiency matrix

\begin{tabular}{|c|c|c|c|c|}
\hline I & II & III & IV & V \\
\hline I & -6 & 3 & 8 & -6 \\
\hline
\end{tabular}

unknowns which reduces to the original five equations when $f_{1} \ldots f_{5}$ are substituted for $u_{1}, u_{2}$ and $u_{3}$. Under these conditions it is not possible to give values for $u_{1}, u_{2}$ and $u_{3}$ which will satisfy the equations exactly, but the maximum likelihood method for estimating these variables will be demonstrated in section 5 .

When information from only duplex parents is available it is easily seen that the same linear combinations of the mode frequencies are 
estimable. The estimation in this case reduces to solving three simultaneous linear equations for the three unknowns. When information from only simplex or triplex parents is available, only the combination I $2 f_{1}+6 f_{2}+3 f_{4}$ or its complement $6 f_{2}+\mathrm{I} 2 f_{3}+9 f_{4}+\mathrm{I} 2 f_{5}$ may be estimated.

TABLE 8

Ordered tetrad matrix for digenic parental genotypes

\begin{tabular}{|c|c|c|c|c|c|c|c|c|c|}
\hline \multicolumn{2}{|c|}{ Parental genotype } & \multicolumn{2}{|c|}{ Simplex } & Aaaa & \multicolumn{4}{|c|}{ Duplex } & \multirow[b]{2}{*}{$\begin{array}{l}\mathrm{Aa}, \mathrm{Aa} ; \\
\mathrm{Aa}, \mathrm{Aa}\end{array}$} \\
\hline $\begin{array}{l}\text { Mod } \\
\text { tetra }\end{array}$ & $\begin{array}{l}\text { Tetrad } \\
\text { genotype }\end{array}$ & $\begin{array}{l}\text { AA, aa; } \\
\text { aa, aa }\end{array}$ & $\begin{array}{l}\mathrm{Aa}, \mathrm{Aa} ; \\
\mathrm{aa}, \mathrm{aa}\end{array}$ & $\begin{array}{l}\mathrm{Aa}, \mathrm{aa} ; \\
\mathrm{Aa}, \mathrm{aa}\end{array}$ & $\begin{array}{c}\mathrm{AA}, \mathrm{AA} ; \\
\text { aa, aa }\end{array}$ & $\begin{array}{l}\text { AA, aa; } \\
\text { AA, aa }\end{array}$ & $\begin{array}{c}\mathrm{AA}, \mathrm{Aa} ; \\
\mathrm{Aa}, \mathrm{aa}\end{array}$ & $\begin{array}{l}\mathrm{AA}, \mathrm{aa} ; \\
\mathrm{Aa}, \mathrm{Aa}\end{array}$ & \\
\hline I & 11,$22 ; 33,44$ & 12 & & $\ldots$ & 4 & 8 & $\ldots$ & & \\
\hline & 11,$22 ; 34,34$ & 6 & 6 & $\ldots$ & $\begin{array}{l}4 \\
4\end{array}$ & ... & $\ldots$ & $\ddot{8}$ & $\cdots$ \\
\hline & II, $34 ; 22,34$ & 6 & $\ldots$ & 6 & ... & 4 & .8 & $\ldots$ & $\ldots$ \\
\hline & 12,$12 ; 34,34$ & $\ldots$ & 12 & $\ldots$ & 4 & $\ldots$ & $\ldots$ & $\ldots$ & 8 \\
\hline & 12,$34 ; 12,34$ & $\ldots$ & $\ldots$ & 12 & $\ldots$ & 4 & $\ldots$ & $\ldots$ & 8 \\
\hline & 11,$23 ; 24,34$ & 3 & 3 & 6 & $\cdots$ & $\ldots$ & 8 & 4 & $\ldots$ \\
\hline & 12,$13 ; 24,34$ & $\ldots$ & 6 & 6 & $\ldots$ & $\ldots$ & 8 & .. & 4 \\
\hline VIII & 12,$34 ; 13,24$ & $\ldots$ & ... & 12 & $\cdots$ & $\ldots$ & $\ldots$ & 8 & 4 \\
\hline
\end{tabular}

Deficiency matrix

\begin{tabular}{|c|c|c|c|c|c|c|c|}
\hline I & II & III & IV & V & VI & VII & VIII \\
\hline I & $\ldots$ & -2 & -1 & $\ldots$ & $\ldots$ & 2 & $\ldots$ \\
$\ldots$ & I & $\ldots$ & -1 & $\ldots$ & -2 & 2 & $\ldots$ \\
$\ldots$ & $\ldots$ & 1 & $\ldots$ & -1 & -2 & 1 & 1 \\
\hline
\end{tabular}

Reduced tetrad matrix

\begin{tabular}{|c|c|c|c|c|c|c|c|c|c|}
\hline $\begin{array}{l}\text { Estimable } \\
\text { mode combinations }\end{array}$ & $\begin{array}{l}\text { Genotype } \\
\text { frequencies }\end{array}$ & $m_{3}$ & $m_{2}$ & $m_{3}$ & $m_{4}$ & $m_{\delta}$ & $m_{6}$ & $m_{7}$ & $m_{\mathrm{s}}$ \\
\hline $\begin{array}{l}u_{1}=f_{1}+f_{1}+f_{2} \\
u_{2}=f_{8}+2 f_{1}+f_{3} \\
u_{3}=f_{6}+4 f_{1}+2 f_{2}+2 f_{3} \\
u_{4}=f_{7}-2 f_{1}-2 f_{2}-f_{3} \\
u_{6}=f_{8}-22_{1}-f_{3} .\end{array}$ & $\begin{array}{l}\cdot \\
: \\
:\end{array}$ & $\begin{array}{l}\cdots \\
\cdots \\
3 \\
\cdots \\
\cdots\end{array}$ & $\begin{array}{r}12 \\
\cdots \\
3 \\
6 \\
\cdots\end{array}$ & $\begin{array}{r}\ldots \\
12 \\
6 \\
6 \\
12\end{array}$ & $\begin{array}{l}4 \\
\cdots \\
\cdots \\
\cdots \\
\cdots\end{array}$ & $\begin{array}{l}\cdots \\
4 \\
\cdots \\
\cdots \\
\cdots\end{array}$ & $\begin{array}{l}\cdots \\
\dddot{8} \\
8 \\
\cdots\end{array}$ & $\begin{array}{c}\cdots \\
\cdots \\
4 \\
\dddot{8}\end{array}$ & $\begin{array}{r}8 \\
8 \\
\cdots \\
4 \\
4\end{array}$ \\
\hline
\end{tabular}

(b) Trigenic parental genotype. The tetrad and deficiency matrix for this case are given in table 7. Four linear combinations of the mode frequencies can be estimated. Moreover the one element of deficiency from the trigenic case is common to the deficiency matrix for a digenic locus so that from combined digenic and trigenic data this element of deficiency still remains. The estimation of the frequencies of all five 
modes of tetrad formation is thus shown to be possible only by using a tetragenic parental genotype.

\section{(ii) Ordered tetrads}

The combined tetrad matrices for simplex and duplex parental genotypes, together with the three-row deficiency matrix and the reduced tetrad matrix for five estimable frequencies are presented in table 8 .

As with unordered tetrads, all estimable linear combinations of the mode frequencies may be estimated from duplex data only. Three independent linear combinations of these parameters may be estimated from simplex or triplex data. However, when $\mathrm{A}$ is dominant to a, the two tetrad genotypes $\mathrm{AA}, \mathrm{Aa} ; \mathrm{Aa}$, aa and $\mathrm{AA}$, aa; $\mathrm{Aa}, \mathrm{Aa}$ arising from duplex parents, and similarly the genotypes $\mathrm{AA}, \mathrm{AA} ; \mathrm{Aa}, \mathrm{Aa}$ and $\mathrm{AA}, \mathrm{Aa}$; $\mathrm{AA}, \mathrm{Aa}$ arising from triplex parents will be phenotypically indistinguishable. If only phenotypically classified tetrad data are available, the relevant columns in the tetrad matrices must be combined, and it is found that in both cases one less combination is estimable.

\section{ESTIMATION OF MODE FREQUENCIES}

(I) Data

Consider tetrad data for the histidine locus in Saccharomyces cerevisice taken from Leupold (1956a). From four parental strains, the following results were obtained:

\begin{tabular}{|c|c|c|}
\hline Strain & Parental genotype & $\begin{array}{c}\text { Phenotype of unordered tetrads } \\
\text { formed }\end{array}$ \\
\hline I & HHhh & I I $(4 \mathrm{H}: o h): 6(3 \mathrm{H}: \mathrm{Ih}):$ I $(2 \mathrm{H}: 2 \mathrm{~h})$ \\
\hline 2 & $\mathrm{HHHh}$ & I $8(4 \mathrm{H}: \mathrm{oh}): \mathrm{o}(3 \mathrm{H}: \mathrm{Ih})$ \\
\hline 3 & Hhhh & $14(2 \mathrm{H}: 2 \mathrm{~h}): 3(\mathrm{H}: 3 \mathrm{~h}): \mathrm{I}(3 \mathrm{H}: \mathrm{Ih})$ \\
\hline 4 & HHhh & $6(4 \mathrm{H}: 0 h):$ I I $(3 \mathrm{H}:$ Ih $): \mathrm{I}(2 \mathrm{H}: 2 \mathrm{~h}$ \\
\hline
\end{tabular}

From data of this kind it is possible in all cases to infer the tetrad genotype from its phenotype, those from strain $I$ for instance being I I $(\mathrm{Hh}, \mathrm{Hh}, \mathrm{Hh}, \mathrm{Hh}): 6(\mathrm{HH}, \mathrm{Hh}, \mathrm{Hh}, \mathrm{hh}): \mathrm{I}(\mathrm{HH}, \mathrm{HH}, \mathrm{hh}, \mathrm{hh})$. The results from strains $\mathrm{I}$ and 4 can be shown to be homogeneous and may be pooled. Making the gene substitution ( $\mathrm{Hh}$ ) as explained in section 4 shows that the data from parent 2 are equivalent to a set of tetrads I $8(\mathrm{Hh}, \mathrm{Hh}, \mathrm{Hh}, \mathrm{hh}): \mathrm{o}(\mathrm{HH}, \mathrm{hh}, \mathrm{hh}, \mathrm{hh})$ from a parent $\mathrm{Hhhh}$, assuming no effects are associated with the gene substitution. The results from strain 3 , ignoring the single $(3 \mathrm{H}: \mathrm{Ih})$ ascus which is inexplicable in terms of a normal meiosis, can be shown to be homogeneous with these results, and the two sets may be pooled. Thus observed frequencies for the five tetrad genotypes detailed in table 4 are $3,32,2,17$ and 17 respectively. 


\section{(ii) Estimation using maximum likelihood}

Since $u_{1}, u_{2}$ and $u_{3}$ are subject to the linear constriction $u_{1}+u_{2}+u_{3}=\mathrm{I}$, the analysis is complicated by the fact that the rows and columns of the covariance matrix of these variables sum to zero, and hence the information matrix will contain infinite elements. This difficulty may be overcome in two ways. The symmetrical approach employed by Fisher (1947) involves substituting new variaables $e_{1}, e_{2}$ and $e_{3}$ defined by the relationships

$$
\frac{d}{d e_{i}}=u_{i}\left\{\frac{d}{d e_{i}}-\sum_{i} u_{i} \frac{d}{d e_{i}}\right\}
$$

where $i=\mathrm{I}, 2,3$ in this case. The method to be used in this paper consists more simply of substituting for one variable in terms of the other two and completing the estimation using these two variables. Although the convergence will in general be faster with a symmetrical substitution, in the case where there are few parameters to estimate the simpler substitution appears to be the more expeditious.

Substituting $u_{2}=\mathrm{I}-u_{1}-u_{3}$ in the expectations given by table 5 , and using the notation that $\mathrm{L}$ represents the logarithm of the likelihood function, we get

$$
\begin{gathered}
\frac{d \mathrm{~L}}{d u_{1}}=-\frac{3}{\mathrm{I}-u_{1}+u_{3}}+\frac{32}{3+u_{1}-u_{3}}-\frac{\mathrm{I} 7}{3-u_{1}-u_{3}}+\frac{17}{u_{1}} \\
\frac{d \mathrm{~L}}{d u_{3}}=\frac{3}{\mathrm{I}-u_{1}+u_{3}}-\frac{32}{3+u_{1}-u_{3}}+\frac{2}{u^{3}}-\frac{\mathrm{I} 7}{3-u_{1}-u_{3}}
\end{gathered}
$$

and similarly we can obtain expressions for

$$
\frac{d^{2} \mathrm{~L}}{d u_{1}^{2}}, \frac{d^{2} \mathrm{~L}}{d u_{1} d u_{3}} \text { and } \frac{d^{2} \mathrm{~L}}{d u_{3}^{2}} .
$$

Using an iterative method, if we take $u_{1}^{0}$ and $u_{3}^{0}$ as first approximations to the true solutions $\hat{u}_{1}$ and $\hat{u}_{3}$, then second approximations $u_{1}^{1}$ and $u_{3}^{1}$ may be found using the equations:

$$
\begin{aligned}
& \left(u_{1}^{1}-u_{1}^{0}\right)\left(\frac{d^{2} \mathrm{~L}}{d u_{1}^{2}}\right)_{u_{1}^{0}, u_{3}^{0}}+\left(u_{3}^{1}-u_{3}^{0}\right)\left(\frac{d^{2} \mathrm{~L}}{d u_{1} d u_{3}}\right)_{u_{1}^{0}, u_{3}^{0}}=-\left(\frac{d \mathrm{~L}}{d u_{1}}\right)_{u_{1}^{0}, u_{3}^{0}} \\
& \left(u_{1}^{1}-u_{1}^{0}\right)\left(\frac{d^{2} \mathrm{~L}}{d u_{1} d u_{3}}\right)_{u_{1}^{0} u_{3}^{0}}+\left(u_{3}^{1}-u_{3}^{0}\right)\left(\frac{d^{2} \mathrm{~L}}{d u_{3}^{2}}\right)_{u_{1}^{0}}, u_{3}^{0}=-\left(\frac{d \mathrm{~L}}{d u_{3}}\right)_{u_{1}^{0}}, u_{3}^{0}
\end{aligned}
$$

$u_{1}^{1}$ and $u_{3}^{1}$ may then be used as the new $u_{1}^{0}$ and $u_{3}^{0}$ from which more accurate approximations may be found. 
In the present case, using the first approximations

then

$$
u_{1}^{0}=\mathrm{I} \cdot \mathrm{I}, \quad u_{3}^{0}=0 \cdot 3
$$

and

$$
\left(\frac{d \mathrm{~L}}{d u_{1}}\right)_{u_{1}^{0}, u_{3}^{0}}=-1 \cdot 7494^{\circ},\left(\frac{d \mathrm{~L}}{d u_{3}}\right)_{u_{1}^{0}, u_{3}^{0}}=2 \cdot 6206 \mathrm{I}
$$

$$
\begin{aligned}
\left(\frac{d^{2} \mathbf{L}}{d u_{1}^{2}}\right)_{u_{1}^{0} u_{3}^{0}}=-97.90628 & \left(\frac{d^{2} \mathrm{~L}}{d u_{1}, d u_{3}}\right)_{u_{1}^{0}}, u_{3}^{0}=70.57544 \\
& \left(\frac{d^{2} \mathbf{L}}{d u_{3}^{2}}\right)_{u_{1}^{0} u_{3}^{0}}=-106.0789 \mathrm{I}
\end{aligned}
$$

Solving the above set of equations yields

$$
u_{1}^{1}=\mathrm{I} \cdot 09989 \quad u_{3}^{1}=0 \cdot 32463
$$

which may then be used as the new $u_{1}^{0}$ and $u_{3}^{0}$. When the process has been carried through two more stages, we nave solutions accurate to five decimal places

$$
\hat{u}_{1}=1 \cdot 09874 \quad \hat{u}_{3}=0.32672
$$

The covariance matrix associated with these variables, which is the inverse of the matrix

comes to

$$
\left(\left\{\frac{d^{2} \mathrm{~L}}{d u_{i} d u_{j}}\right\}_{\hat{u}_{1} \hat{u}_{3}}\right)
$$

$$
\begin{array}{ll}
0.020860 & 0.012949 \\
0.012949 & 0.019725
\end{array}
$$

$u_{2}$ is now obtained as $\mathrm{I}-\hat{u}_{1}-\hat{u}_{3}=-0.42546$.

Using the property that the rows and columns of the covariance matrix of $u_{1}, u_{2}$ and $u_{3}$ add to zero, we may obtain the remaining elements, giving

$$
\begin{array}{rrr}
0.020860 & -0.033^{809} & 0.012949 \\
-0.033809 & 0.066483 & -0.032674 \\
0.012949 & -0.03^{26} 674 & 0.019725
\end{array}
$$

\section{ESTIMABLE PARAMETERS}

(i) Unordered tetrads

From single locus random spore data from a tetrasomic organism only one parameter, the frequency of double reduction can be estimated. In terms of the frequencies of the modes of tetrad formation $a$ is evidently $f_{1}+\frac{1}{2} f_{2}+\frac{1}{4} f_{4}$, which can easily be shown to be estimable from tetrad data from all parental genotypes. In terms of $u_{1}, u_{2}$ and $u_{3}$ defined in table $4, a$ is equal to $\frac{1}{4} u_{2}+\frac{1}{2} u_{3}$. Substituting the values of $u_{2}$ and $u_{3}$ found from data in section 5

$$
a=0.056 \text {. }
$$


Variance of $\alpha=\frac{1}{16} \mathrm{~V}\left(u_{2}\right)+\frac{1}{4} \operatorname{Cov}\left(u_{2} u_{3}\right)+\frac{1}{4} \mathrm{~V}\left(u_{3}\right)$ giving $\mathrm{V}(\alpha)=0 \cdot 00092$, or a standard deviation for the above estimate of 0.030 .

\section{(ii) Ordered tetrads}

The frequency of double reduction can be shown to be estimable from simplex, duplex and triplex data, whether or not back-crossing to identify all genotypes has been carried out. For example, in terms of the set of $u$ 's defined in table 8:

$$
a=f_{1}+\frac{1}{2} f_{2}+\frac{1}{2} f_{3}+\frac{1}{4} f_{6}=\frac{1}{4} u_{3} .
$$

Apart from the frequency of double reduction it is difficult to determine by inspection which linear combinations of the mode frequencies give information on the position of the locus or the type of chromosome configuration involved. The principal reason for this difficulty lies

TABLE 9

Matrix for mode frequencies in terms of interphase mode frequencies

\begin{tabular}{|c|c|c|c|c|c|c|c|c|c|}
\hline & hase modes & \multicolumn{3}{|c|}{ Interphase modes } & IV & & VI & VII & VIII \\
\hline$I^{\prime}$ & 11,$22 ; 33,44$ & $\cdots$ & $\cdots$ & $\ldots$ & 4 & $\cdots$ & $\cdots$ & $\cdots$ & $\cdots$ \\
\hline II' $^{\prime}$ & 11,$22 ; 34,34$ & $\ldots$ & 2 & $\ldots$ & 2 & $\ldots$ & $\ldots$ & $\ldots$ & $\ldots$ \\
\hline III' & 11,$34 ; 22,34$ & $\cdots$ & $\cdots$ & $\ldots$ & $\ldots$ & $\cdots$ & $\cdots$ & 4 & $\cdots$ \\
\hline IV' & 12,$12 ; 34,34$ & 1 & 2 & $\cdots$ & I & $\cdots$ & $\cdots$ & $\cdots$ & $\cdots$ \\
\hline $\mathrm{V}^{\prime}$ & 12,$34 ; 12,34$ & ... & $\ldots$ & $\ldots$ & $\ldots$ & 2 & $\ldots$ & $\cdots$ & 2 \\
\hline $\mathrm{VI}^{\prime}$ & II, $23 ; 24,34$ & $\ldots$ & $\ldots$ & $\ldots$ & $\ldots$ & $\ldots$ & 2 & 2 & ... \\
\hline VII' & 12,$13 ; 24,34$ & $\ldots$ & $\ldots$ & I & $\ldots$ & ... & 2 & I & $\cdots$ \\
\hline VIII' $^{\prime}$ & $\mathrm{I} 2,34 ; 13,24$ & $\cdots$ & $\cdots$ & $\cdots$ & $\cdots$ & 2 & $\cdots$ & $\cdots$ & 2 \\
\hline
\end{tabular}

in the fact that the chromosome configuration given by recombination and disjunction at the first division of meiosis is to some extent broken up by the second division of meiosis. For this reason it is at times more revealing to deal with the gene configuration before the second division of meiosis.

At the locus in question, we may consider the gene configuration with regard to the four centromeres at the interphase stage of meiosis. The same eight configuration types are possible at this stage as at the final tetrad stage, the notation being again that the semi-colon separates the products of the first division of meiosis. These types, which may be called interphase modes, will be denoted as $\mathrm{I}^{\prime}, \mathrm{II}^{\prime}, \ldots$ VIII' as shown in table 9 , with frequencies $f_{1}^{\prime}, f_{2}^{\prime}, \ldots f_{8}^{\prime}$. The concept of the interphase mode as considered here is similar to that of the "quadrivalent type" of Leupold ( $1956 b)$ where the distribution is considered with regard to the four centromeres before chromosome disjunction. However consideration of the configuration at the later stage of meiosis avoids the need for assumptions about the type of chromosome disjunction.

It is necessary at this stage to define the relationships between the mode and interphase mode frequencies. The obvious assumption to 
be made is that of random orientation of chromatids on the metaphase plate at the second division of meiosis. Under this assumption the matrix connecting the mode and interphase mode frequencies may be drawn up as in table 9. Although the assumption does not appear to be very restrictive, it may not be accurate at all times (Whitehouse and Haldane, 1946). However it is felt that small departures from randomness would not greatly affect the argument.

Of the eight equations giving $f_{1} \ldots f_{8}$ in terms of $f_{1}^{\prime} \ldots f_{8}^{\prime}$ in table 9 only seven are independent. The frequencies $f_{5}^{\prime}$ and $f_{8}^{\prime}$ are not individually expressible in terms of $f_{1} \ldots f_{8}$ but the sum $f_{5}^{\prime}+f_{8}^{\prime}$ is equal to $f_{5}+f_{8}$. The equations for the remaining $f^{\prime \prime}$ s are

$$
\begin{array}{ll}
f_{1}^{\prime}=f_{1}-f_{2}+f_{4} & f_{3}^{\prime}=f_{3}-f_{6}+f_{7} \\
f_{2}^{\prime}=-4 f_{1}+2 f_{2} & f_{6}^{\prime}=-4 f_{3}+2 f_{6} \\
f_{4}^{\prime}=4 f_{1} & f_{7}^{\prime}=4 f_{3}
\end{array}
$$

A matrix may now be drawn up connecting the eight possible tetrad genotypes to the interphase modes. If the matrices of tables 8 and 9 are denoted as $\mathrm{G}$ and $\mathrm{M}$, then the equations represented by these tables may be written in matrix form as

$$
f \mathrm{G}=m \text { and } f^{\prime} \mathrm{M}=f .
$$

Therefore $f^{\prime} \mathrm{MG}=f \mathrm{G}=m$, giving the matrix connecting the genotypes and interphase modes as MG. This matrix, and the associated three-row deficiency matrix are given in table 10. Postmultiplying the rows of the deficiency matrix of table ro by $M$, the first two rows of the deficiency matrices associated with $G$ and $M G$ are found to be equal, but this is not true for the third rows. Thus we are led

TABLE 10

Matrix for genotype frequencies in terms of interphase mode frequencies

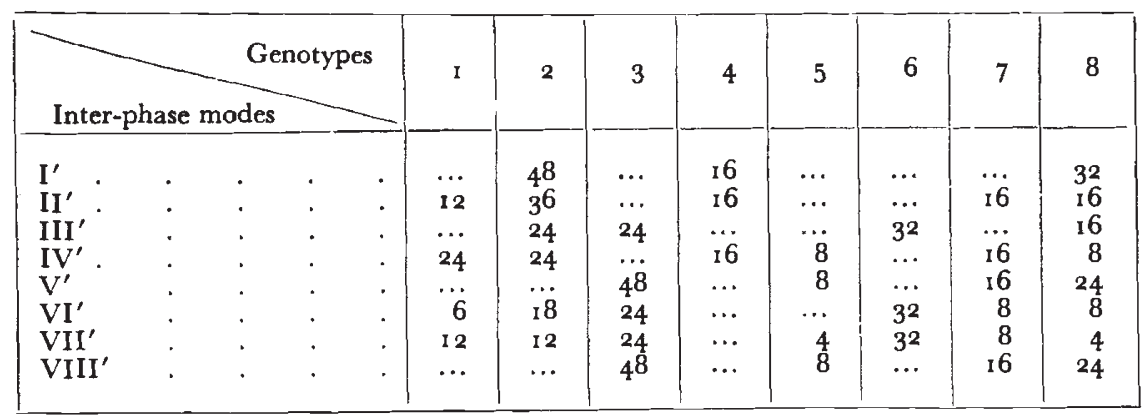

Deficiency matrix

\begin{tabular}{|c|c|c|c|c|c|c|c|}
\hline $\mathrm{I}^{\prime}$ & $\mathrm{II}^{\prime}$ & $\mathrm{III}^{\prime}$ & $\mathrm{IV}^{\prime}$ & $\mathrm{V}^{\prime}$ & $\mathrm{VI}^{\prime}$ & $\mathrm{VII}^{\prime}$ & $\mathrm{VIII}^{\prime}$ \\
\hline$\ldots$ & $-\mathrm{I}$ & $\ldots$ & $\mathrm{I}$ & $\ldots$ & 2 & -2 & $\ldots$ \\
$-\mathrm{I}$ & $\mathrm{I}$ & 2 & $\ldots$ & $\cdots$ & -2 & $\ldots$ & $\ldots$ \\
$\cdots$ & $\cdots$ & $\cdots$ & $\cdots$ & $\mathrm{I}$ & $\cdots$ & $\cdots$ & $-\mathrm{I}$ \\
\hline
\end{tabular}


to the conclusion that certain combinations of the mode frequencies although previously not estimable, can be estimated when the assumption of random orientation of chromatids is taken into account. The reason for this lies in the fact that the expected frequencies of modes $\mathrm{V}$ and VIII are now equal as can be seen from table 9. A linear combination of the mode frequencies which premultiplies rows one and two but not row three of the deficiency matrix of table 8 to zero, and which is therefore a linear combination that is not estimable, may be chosen so as to premultiply row three to zero by adding suitable multiples of $\left(f_{5}-f_{8}\right)$. The combination $2 f_{2}-f_{3}+2 f_{4}+2 f_{5}$ for instance is equal in expected value to $2 f_{2}-f_{3}+2 f_{4}+2 f_{5}-\frac{3}{2}\left(f_{5}-f_{8}\right)$ which is an estimable combination of the mode frequencies. The third element of deficiency of the matrix $G$ thus disappears under the assumption considered, but is replaced by the element $f_{5}^{\prime}-\mathrm{f}_{8}^{\prime}$ in the matrix $M G$, since the relative contribution of interphase modes $V^{\prime}$ and VIII' to modes V and VIII cannot be determined.

The principal use of the interphase modes can be illustrated in the derivation of a parameter providing an estimate of the locus-centromere distance. It may be seen by inspection that the sum $f_{1}^{\prime}+\frac{1}{2} f_{2}^{\prime}+\frac{1}{2} f_{3}^{\prime}+\frac{1}{4} f_{6}^{\prime}=\mathrm{I}-e$ gives the frequency with which no chiasma occurs between the locus and the centromere, assuming that no double crossovers have occurred. Expressing $\mathrm{I}-e$ in terms of the mode frequencies gives $-f_{1}-\frac{1}{2} f_{2}+f_{4}+\frac{1}{2} f_{7}$, which is estimable from simplex tetrad data, or genotypically classified duplex or triplex tetrad data. Corresponding to the relationship-map distance of locus from centromere $=\frac{1}{2} \times$ frequency of second division segregation, for tetrads from diploid organisms, the relationship-map distance $=\frac{1}{2} \times e$ can be given for tetraploids. The relationship may in fact be accurate for longer distances in tetraploids where, with multivalent formation, a higher proportion of double crossovers are likely to involve three or four strands than is the case in diploids.

While the frequency of double reduction is dependent on the frequency of quadrivalent formation, the parameter $e$ is independent of both the type of chromosome configuration and disjunction. Nevertheless we cannot immediately obtain an estimate of the frequency of quadrivalent formation $q$, from the parameters $e$ and $a$ since $q$ is associated with a parameter measuring the frequency of one type of chromosomal disjunction. Under the assumption of random chromosome segregation, i.e., any two of the four centromeres are equally likely to disjoin together, this parameter has the value one third, and the estimate of $q$ becomes $\frac{6 a}{e}$. Unfortunately it would be extremely difficult to test genetically the validity of the assumption of random chromosome segregation even if fully classified tetrad data were available. This is because when $q$ occurs in the expectations of mode frequencies based on simple models, it is always compounded with the above-mentioned segregation parameter. 
Genetical evidence for multivalent formation may be obtained by the recognition of modes I, II, III and VI, since none of these modes may be produced by bivalents. The frequency $f_{1}+f_{2}+f_{3}+f_{6}$ may therefore be given as a lower bound for $q$. This frequency however is not estimable from digenic tetrad data, and it may readily be seen from the deficiency matrix of table 8 that the greatest estimable lower bound is $f_{1}+\frac{1}{2} f_{2}+\frac{1}{2} f_{3}+\frac{1}{4} f_{6}$, which equals the frequency of double reduction. Comparison of this value with the estimated value of $q$ of $\frac{6 a}{e}$ obtained above shows that this lower bound is likely to be considerably below the true value of $q$. An improved lower bound may be obtained by consideration of the interphase modes, but this value could be suspect since it relies on the assumption of random orientation of chromatids in meiosis.

It should be noted that use of the concept of interphase modes is not restricted in principle to ordered tetrads. Since the process of formation of unordered tetrads is the same during meiosis, the equivalent table to table io for unordered tetrads is obtained by pooling columns 2 and 3,4 and 5, and 6 and 7 . From the $8 \times 5$ matrix formed however it may be seen that only three independent parameters may be estimated. The parameter $e$, for instance, is not estimable from this type of data.

\section{SUMMARY}

Methods used by Fisher (1947) in the analysis of gametic data from a tetrasomic organism have been adapted for use in the analysis of single locus tetrad data in tetrasomic inheritance. Data of this kind have been analysed previously by Roman et al. (1956) and Leupold ( $1956 a$ ), but these results were obtained utilising limiting assumptions of random chromosome segregation and either bivalent formation, simple quadrivalent formation, or quadrivalent formation allowing random partner exchange. The analysis carried out in the present paper is directed at estimating a different set of parameters from those considered by the above authors, for which no assumptions of the above type need be made.

Enumeration formulæ developed for the case of tetrad formation in tetrasomic organisms by Fisher (1950) and James and Papazian (I96I) are discussed, and further enumerations relevant to the methods of this paper are given. The concepts of mode of tetrad formation and tetrad genotype are defined and illustrated by reference to a single tetrasomic locus, and the relationships between the mode frequencies and observable genotype frequencies are calculated for ordered and unordered tetrads. The estimation of the mode frequencies is then discussed, and illustrated for unordered tetrads by the analysis of published data of autotetraploid yeast. It is shown that more precise information may be obtained from ordered tetrad data than from 
unordered tetrad data if the assumption of random orientation of chromatids at the second division of meiosis is utilised. A parameter analogous to the frequency of second division segregation in diploids may then be estimated, and also estimates obtained of the frequency of quadrivalent formation.

Acknowledgments. - The author would like to thank Professor J. H. Bennett for his assistance and criticism at all stages in the preparation of this paper. Portion of the work was done during the tenure of a C.S.I.R.O. senior studentship.

\section{REFERENCES}

BENNETt, J. H. 1956. Modes of tetrad formation. American Naturalist, 90, 195199.

FISHER, R. A. 1947. The theory of linkage in polysomic inheritance. Phil. Trans. Roy. Soc. B, 233, 55-87.

FISHER, R. A. 1950. A class of enumerations of importance in genetics. Proc. Roy. Soc. $B, 136,509-520$.

FISHER, R. A. 1962. Enumeration and classification in polysomic inheritance. 7. Theoretical Biology, 2, 309-311.

GRIFFING, B. 1957. Linkage in trisomic inheritance. Heredity, $I 1,69-92$.

JAMES, A. T., AND PAPAZIAN, H. P. 1961. Enumeration of quad types in diploids and tetraploids. Genetics, $46,817-829$.

LEUPOLD, U. 1956a. Tetraploid inheritance in Saccharomyces. Journal of Genetics, $54,411-426$.

LEUPOLD, $U$. 1956b. Tetrad analysis of segregation in autotetraploids. Fournal of Genetics, 54, 427-439.

ROMAN, H., PHILLIPS, M. M., AND SANDS, s. M. 1955. Studies of polyploid Saccharomyces. I. Tetraploid segregation. Genetics, 40, 546-56r.

WHITEHOUSE, H. L. K., AND HALDANE, J. B. S. 1946. Symmetrical and asymmetrical reduction in ascomycetes. Journal of Genetics, 47, 208-2 12. 